\title{
Avaliação econômica em Saúde na esfera de atenção local à saúde
}

\author{
Health economic evaluation in a local level government \\ health care system
}

Leyla Gomes Sancho ${ }^{1}$

José M uniz Costa Vargens ${ }^{2}$

${ }^{1}$ Secretaria de Estado da Saúde de M inas Gerais. Rua Sapucaí, Floresta. 30150-050 Belo Horizonte M G.

Isancho@uol.com.br

${ }^{2}$ Fundação Escola N acional deSeguros.
Abstract This work aims to contribute to the discussion about the possibility of applying health economic evaluations at local level government healthcare system, and consequently use the results of this study into decision making. In order to subside this reflexion, it was analyzed the SAM U/192 Program costs evaluation in the city of Belo H orizonte, as well as data concerning effectiveness of the program and a review on electronic databases (SCiELO and M edline) about the application of studies in decision making. The analysis showed that even for a simple evaluation on expenditure, there are still unsolved problems of data availability as well as of data effectiveness on information systems definition and association. It showed that decision makers do not use the result of studies for decision making either. So, we conclude that there is no possi bility to apply a health economic evaluation research and have the results used in a local level government health care system. Key words Health economic evaluation, Local level government healthcare system, Cost-effectiveness analysis, Decision maker, Decision making
Resumo 0 estudo tem por finalidade contribuir com a reflexão sobre a possibilidade de se operacionalizar avaliações econômicas em saúde nas esferas de atenção local à saúde e a consequente apreensão dos resultados de estudos para efeito de decisão pelos gestores. Para subsidiar essa reflexão, seutilizou como objeto de análise a avaliação realizada sobre o custo do Programa SAM U/192 no município de Belo Horizonte (M G), dados relativos à efetividade do programa e uma revisão nas bases eletrônicas de dados (SciELO eM edline) sobreo uso de estudos para efeito de decisão. A análise apontou que, mesmo para uma simples avaliação de gastos, ainda existem problemas relativos à disponibilidade de dados, como também no que concerne aos dados para subsidiar a avaliação sobre sua efetividade, e em relação à definição de sistemas e o relacionamento entre sistemas de informação. Assim como mostrou que os estudos são pouco utilizados pel os gestores, levando à conclusão de que a condução e a utilização, para efeito de gestão, de estudos deste porte ainda são pouco factíveis nesta esfera de governo.

Palavras-chave Avaliação econômica em saúde, Esfera de atenção local à saúde, Avaliação de custoefetividade, Gestor de sistema de saúde, Tomada de decisão 
Introdução

A avaliação econômica em saúde tem como principal objetivo apoiar tomadas de decisão de forma socialmente eficiente, considerando que as escoIhas, diante a incerteza afeita ao setor saúde e sob menor probabilidade de risco, contribuam para uma repartição mais equânime das intervenções/ ações de saúde e, por decorrência, gerem efetivos ganhos em saúde.

A importância da avaliação econômica em saúde se fundamenta em evidências advindas de diferentes matizes; a econômica, quetem como princípio básico a escassez de recursos diante das necessidades, e a médica (ou melhor, da indústria atrelada aos equipamentos e insumos médicos - diagnóstico, propedêutico e terapêutico), que, a partir da inovação tecnológica, cria crescente demanda por bens e serviçose, consequentemente, por alocação maior de recursos no setor.

A decisão quanto à escolha entre intervenções ou cursos alternativos de uma mesma intervenção ou do conjunto de intervenções relativas a um determinado programa é resultado da utilização de diferentes técnicas analíticas- todas calcadas no enfoque da avaliação microeconômica. As técnicas ditas completas são o custo-minimização, o custobenefício, o custo- efetividade e o custo-utilidade, e a parcial, o custo- consequência. $E$, independente do tipo de análise, todas têm em comum a relação entre o montante de recursos aplicados vis-à-vis os ganhos em saúde decorrentes dessa aplicação.

No Brasil, a avaliação econômica em saúde se encontra em patamar deficitário, seja do ponto de vista da utilização do instrumental, como em relação à condução de estudos. M esmo que agências governamentais de regulação, como a Agência Nacional de Vigilância Sanitária (ANVISA) e a Agência N acional de Saúde Suplementar (ANS), bem como o próprio M inistério da Saúde, por meio de sua política de pagamentos e de alocação de recursos, venham, nos últimos anos, desenvolvendo estudos na área, principalmente os de custo-efetividade?.

0 grande desafio, no entanto, é a extensão da utilização e o desenvolvimento desses estudos no nível operacional de atenção à saúde - os municípios. Isto porque os gestores não percebem a importância do instrumental como um indicativo para uma alocação de recursos de forma eficiente, assim como para a avaliação da efetividade de tecnologias médicas- sanitárias ou como suportepara a regulação de ações de saúde e, principalmente, por não o incorporar como um instrumento complementar às suas decisões. A não incorporação do instrumental pode ser explicada pela (in) formação insuficiente quanto à validade desse instrumental para tomada de decisão e/ou como consequência da divulgação ainda inci pientedeestudos sobre avaliação econômica, ou mesmo por priorizar a alocação sob o jugo político, em vista do dilema existente entre a ousadia de inovar nos critérios decisórios e o pragmatismo da continuidade dos critérios que favorecem a permanência do status quo.

Outro desafio, e independente sob qual esfera de governo, é a padronização metodológica à consecução desses estudos. Garber, já no ano de 1999, em seu estudo Advances in Cost-Effectiveness Analysis of $\mathrm{H}$ ealth Intervention, como um avanço sobre as suas próprias reflexões, chamava a atenção para os esforços em relação à padronização e a conduta metodológica, realizados em diversos países, citando inclusive vários guias elaborados, como 0 Australian Pharmaeconomics, o European Community Concerted Action on the $\mathrm{H}$ armonisation of the $M$ ethodology for Economic Evaluation of $\mathrm{Health}$ Technology (HARM ET) e o Panel on Cost-Effectiveness in $\mathrm{H}$ ealth and M edicine ${ }^{2}$.

No entanto, só recentementeno Brasil, em 2007, a partir de iniciativa do Ministério da Saúde, se formalizou um manual de diretrizes metodológicas para a execução de avaliações econômicas ${ }^{3}$ e um manual teórico sobre avaliação econômica ${ }^{4}$, os quais ainda não foram devidamente avaliados quanto a sua disseminação e adoção.

Para, no entanto, desenvolver estudos deste porte - independente se a partir de uma vertente epidemiológica (ensaios clínicos e outros tipos de estudo) ou matemática (modelagem probabilística) -, éimprescindível que os dados estejam disponíveis. Tanto os relativos ao custo, como aqueles relativos à morbimortalidade e/ou em relação a outras informações que subsidiem a construção demedidas ou índices de interesse para a avaliação da efetividade de uma determinada interven ção e/ ou programa.

A Secretaria Municipal de Saúde de Belo Horizonte (SM SA-BH), no período compreendido entre 1998 e 2005, teve incorporado em sua estrutura organizacional um setor de apuração de custos, cujo objetivo era implantar sistemas de informação em custos nas unidades de produção (unidades básicas, unidades de urgências e policlínicas) e realizar estudos pontuais sobre avaliação de gastos em situações-problemas. 0 objetivo foi logrado parcialmente devido a problemas ora decunho operacional, ora político.

$\mathrm{N}$ a perspectiva do exposto, ediante do cenário encontrado na SM SA-BH no que tange à restrita 
disponibilidade de dados - até para uma simples avaliação de alocação de recursos - , e em relação à qualidade dos dados e à especificação e relacionamento dos sistemas de informação na instituição, se processou uma reflexão sobre a viabilidade da condução de estudos sobre avaliação econômica em saúde na realidade do dia-a-dia dos municípios e a decorrente apreensão de seus resultados pelos gestores de sistemas de saúde para efeito de decisão.

\section{M etodologia}

A reflexão que se propõe este estudo foi pautada a partir de uma análise sobre a viabilidade de condução de estudos de custo-efetividadeno nível local de atenção à saúde, a possibilidade de construção de medidas de efetividadea partir de dados einformações disponíveis nos sistemas locais de informação e a utilização da ferramenta pelos gestores.

A viabilidade de condução de estudos foi realizada a partir do estudo, tomado como objeto de análise, sobre o custo do Programa SAMU/192 realizado pelo setor de custos da Gerência Administrativa da SM SA-BH, no ano de 2004, no município de Belo Horizonte (M G) -, no qual se procurou avaliar inicialmente a metodologia utilizada e sob qual perspectiva o estudo foi conduzido. Seguida pela avaliação da sua operacional ização, em termos de captação dos dados, da mensuração e valoração dos custos e quais as dificuldades encontradas para a efetivação desses procedimentos.

A construção de medidas de efeito/efetividade (indicadores) foi analisada através de uma averiguação do conteúdo das informações existentes no banco de dados do setor responsável (Gerência de Urgência e Emergência) pela coordenação do programa, na qual se buscou identificar a sistematização das variáveis utilizadas - e a decorrente coleta de dados - e se essas variáveis seriam suficientes para conduzir uma avaliação sobre efetividade.

A utilização da ferramenta pelos gestores para efeito de decisão foi verificada através deuma busca por estudos, que contemplassem esse objetivo, na base eletrônica de dados SciELO eM edline, entreos anos de 2005 e 2007, por meio das seguintes palavras-chave: cost-effectiveness, cost-effectiveness and Brazil, cost-effectivenessand Brazil and decision maker, cost-effectiveness and decision maker, cost-effectiveness and decision maker and health-care, cost-effectiveness and decision maker and healthcare, cost-effectiveness and decision maker and healthcare system e cost-effectiveness and healthcare system.
Viabilidade da condução de estudos a partir da análise de um estudo-custo do Programa SAM U/192

No quese refereà viabilidade de condução deestudos no nível local - tendo como exemplo o estudo realizado sobre o custo do Programa SAM U - a análise preliminar identificou que a metodologia adotada nesse estudo foi a do custeio direto e sua consecução sob a perspectiva do governo, em que somente os custos diretos e os de overhead foram contabilizados.

A sua operacionalização privilegiou, por questões pragmáticas, ora os dados sob a concepção de custos, ora sob a de gasto. 0 fato é decorrente da não contabilização do custo a partir do efetivo consumo de insumos, pela ausência de informação quanto ao número de horas trabalhadas pelos recursos humanos por atendimento pré-hospitalar prestado, pela falta de informação quanto ao tipo e número de horas utilizadas de serviços de manutenção pelas bases operacionais, ou mesmo pela inexistência de dados, conforme demonstrado no Quadro 1. Ou seja, a composição do custo foi elaborada ora sob um critério linear - por exemplo, os relativos à manutenção - , ora em termos absolutos - por exemplo, os relativos aos vencimentos dos recursos humanos, e na perspectiva do gasto e não do efetivo consumo - por exemplo, os relativos aos materiais médico-cirúrgico. Vale lembrar que a diferença entreas concepções, na vertente contábil, se assenta na utilização da val oração a partir do efetivo consumo (custo) e a partir do sacrifício financeiro em um determinado período (gasto) ${ }^{5}$.

Assim como vale ressaltar que, em uma avaliação de custo-efetividade na vertente econômica, e na perspectiva do custo-efetividade, o custo unitário (custo total médio) se refere a uma função que mede a relação entre os preços dos fatores de produção e o número de unidades produzidas e tem no custo marginal, que avalia a variação nos custos para uma dada variação no número de unidades produzidas, o resultado de interesse para uma avaliação econômica ${ }^{6}$.

\section{Construção de medidas de efetividade} a partir de bancos de dados

No que concerne à construção de medidas de efetividade, o banco de dados da Gerência de Urgência e Emergência está formatado na perspectiva administrativa, em que os dados (e variáveis) de interesse são o cadastro (nome, endereço, idade, gênero, grupo étnico, data do atendimento), a conduta efetuada ea hi pótese diagnóstica referente ao 
Quadro 1. Custo do Programa SAM U/192 da Secretaria M unicipal de Saúde de Belo Horizonte, 2004.

\begin{tabular}{|c|c|c|c|c|c|c|}
\hline Itens & Gerência & Almoxarifado & $\begin{array}{l}\text { Central de } \\
\text { Regulação }\end{array}$ & $\begin{array}{c}\text { A mbulância } \\
\text { Vip }\end{array}$ & $\begin{array}{c}\text { Base } \\
\text { Barreiro }\end{array}$ & $\begin{array}{c}\text { Base } \\
\text { Betânia }\end{array}$ \\
\hline $\begin{array}{l}\text { Recursos Humanos } \\
\text { Efetivo } \\
\text { Contratados } \\
\text { Terceirizados } \\
\text { Total }\end{array}$ & $\begin{array}{r}12.843,2 \\
1.332,0 \\
5.182,2 \\
19.367,5\end{array}$ & $\begin{array}{r}1.332,0 \\
667,0 \\
869,8 \\
2.868,8\end{array}$ & $\begin{array}{r}9.307,9 \\
11.109,9 \\
32.875,2 \\
53.293,0\end{array}$ & $\begin{array}{r}0,0 \\
9.200,0 \\
0,0 \\
9.200,0\end{array}$ & $\begin{array}{r}5.687,6 \\
2.057,0 \\
- \\
7.744,6\end{array}$ & $\begin{array}{r}10.383,5 \\
15.802,8 \\
- \\
26.186,5\end{array}$ \\
\hline $\begin{array}{l}\text { Encargos sociais dos efetivos ( } 23 \% \text { ) } \\
\text { Energia / água - Cemig/ Copasa } \\
\text { Comunicação - Telemar } \\
\text { Total }\end{array}$ & $\begin{array}{r}2.953,9 \\
- \\
804,0 \\
804,0\end{array}$ & $\begin{array}{r}306,4 \\
- \\
- \\
-\end{array}$ & $\begin{array}{r}2.140,8 \\
- \\
1.219,4 \\
1.219,4\end{array}$ & $\begin{array}{r}0,0 \\
- \\
- \\
-\end{array}$ & $\begin{array}{r}1.308,2 \\
- \\
-\end{array}$ & $\begin{array}{r}2.388,2 \\
- \\
- \\
-\end{array}$ \\
\hline Alimentação & 73,5 & - & - & - & 735,2 & 753,4 \\
\hline $\begin{array}{l}\text { Almoxarifado } \\
\text { M aterial consumo } \\
\text { Depreciação } \\
\text { Total }\end{array}$ & $\begin{array}{l}752,7 \\
233,3 \\
984,9\end{array}$ & $\begin{array}{r}124,9 \\
28.416,2 \\
28.541,2\end{array}$ & $\begin{array}{r}343,3 \\
935,6 \\
1.378,8\end{array}$ & $\begin{array}{r}667,9 \\
1.924,2 \\
2.564,1\end{array}$ & $\begin{array}{l}1.019,2 \\
1.958,7 \\
2.978,0\end{array}$ & $\begin{array}{r}- \\
2.210,0 \\
2.210,0\end{array}$ \\
\hline $\begin{array}{l}\text { M anutenção } \\
\text { oximil } \\
\text { trade } \\
\text { aluguel }\end{array}$ & $\begin{array}{r}0,0 \\
0,0 \\
2.320,8\end{array}$ & $\begin{array}{r}0,0 \\
197.600,0 \\
-\end{array}$ & $\begin{array}{r}0,0 \\
0,0 \\
42,9\end{array}$ & $\begin{array}{r}162,6 \\
8.509,1 \\
42,9\end{array}$ & $\begin{array}{r}162,6 \\
8.509,1 \\
42,9\end{array}$ & $\begin{array}{r}162,6 \\
8.509,1 \\
42,9\end{array}$ \\
\hline Total geral & $26.431,1$ & $229.040,4$ & $58.074,9$ & $20.478,6$ & $21.480,6$ & $40.252,8$ \\
\hline Produção & & & & 8 & 330 & - \\
\hline Custo unitário & & & & $2.559,8$ & 65,9 & - \\
\hline
\end{tabular}

paciente e dados de produção (diário e consolidado mensal). N ão há informações cruzadas quanto à evolução dos pacientes atendidos. Ou seja, não são coletadas informações adicionais dos pacientes atendidos pelo SAMU / 192 nos estabelecimentos (em geral, hospital público) para os quais são encaminhados. Sugerindo que não há informações suficientes para a construção de medidas (indicadores) que avaliem a efetividade da atenção, sejam de cunho intermediário ou final, tais como número de mortes evitadas, diminuição de tempo médio depermanência hospitalar, anos de vida ganho ou mesmo anos de vida ganho ajustados pela qualidade (QALYs ganhos).

Ressalva-se, como adendo, que a efetividade, fundamentada na concepção epidemiológica, é o resultado observado em relação a uma determinada intervenção/tecnologia/programa; ou melhor, uma estimativa numérica da magnitude dos efeitos da intervenção na saúde ${ }^{7}$. Que, à luz de uma avaliação econômica, éum atributo para uma ava- liação de eficiência comparativå . A eficiência comparativa é a síntese de uma avaliação de custoefetividade, ou seja, a relação incremental de custo-efetividade - definida como o produto entre 0 custo adicional eo benefício adicional alcançado $0^{9,10}$.

O gestor de sistema de saú de e estudos de aval iação econômica para tomada de decisão

No que tange à revisão eletrônica sobre a utilização da ferramenta, ficou patente que os últimos anos estudos sobre avaliação econômica em saúde vêm ganhando popularidade nos países desenvolvidos ${ }^{11}$. Ao contrário do que se averiguou, conformea busca, no Brasil, onde o número de estudos é ainda insuficiente, principalmente os relativos à discussão sobre gestor de sistema de saúde e tomada de decisão. 0 resultado da busca se encontra no Quadro 2. 
Quadro 1. continuação

\begin{tabular}{|c|c|c|c|c|c|c|c|}
\hline $\begin{array}{c}\text { Base } \\
\text { Centro-Sul }\end{array}$ & Base Leste & $\begin{array}{c}\text { Base } \\
\text { Nordeste }\end{array}$ & $\begin{array}{c}\text { Base } \\
\text { Noroeste }\end{array}$ & Base PBH & $\begin{array}{l}\text { Base Venda } \\
\text { Nova }\end{array}$ & $\begin{array}{l}\text { Base Venda } \\
\text { Nova II }\end{array}$ & $\begin{array}{c}\text { Base HOB } \\
\text { (Hospital Municipal) }\end{array}$ \\
\hline $27.002,0$ & - & $3.663,3$ & $4.261,5$ & $4.877,5$ & & $19.015,2$ & - \\
\hline $13.136,8$ & $4.781,0$ & $2.724,0$ & $2.724,0$ & $2.001,0$ & $5.420,0$ & $12.441,8$ & $4.809,0$ \\
\hline 927,4 & & - & & & & & . \\
\hline $41.066,3$ & $4.781,0$ & $6.387,3$ & $6.985,5$ & $6.878,1$ & $5.420,0$ & $31.457,1$ & $4.809,0$ \\
\hline $6.210,5$ & - & 842,6 & 980,2 & $1.121,8$ & - & $4.373,5$ & - \\
\hline- & - & - & . & - & - & - & - \\
\hline 42,3 & - & 32,8 & - & - & - & 29,2 & - \\
\hline 42,3 & - & 32,8 & - & - & - & 29,2 & - \\
\hline $1.414,9$ & 514,5 & 661,5 & 735,0 & 735,0 & 588,0 & 900,4 & 514,5 \\
\hline 352,4 & $1.159,4$ & $1.105,0$ & $1.209,0$ & $1.224,7$ & 983,9 & 224,1 & 277,3 \\
\hline $2.256,1$ & $1.962,2$ & $1.957,2$ & $1.919,8$ & $1.926,5$ & $1.955,5$ & $2.200,1$ & $1.914,8$ \\
\hline $2.608,6$ & $3.121,6$ & $3.062,2$ & $3.129,1$ & $3.151,2$ & $2.939,4$ & $2.424,3$ & $2.192,2$ \\
\hline 162,6 & 162,6 & 162,6 & 162,6 & 162,6 & 162,6 & 162,6 & 162,6 \\
\hline $8.509,1$ & $8.509,1$ & $8.509,1$ & $8.509,1$ & $8.509,1$ & $8.509,1$ & $8.509,1$ & $8.509,1$ \\
\hline 42,9 & 42,9 & 42,9 & 42,9 & 42,9 & 42,9 & 42,9 & 42,9 \\
\hline $60.057,2$ & $17.131,7$ & $19.701,0$ & $20.544,4$ & $20.600,7$ & $17.662,0$ & $47.899,1$ & $16.230,3$ \\
\hline 220 & 388 & 420 & 437 & 440 & 397 & 189 & 451 \\
\hline 272,9 & 44,2 & 46,9 & 47,0 & 46,8 & 44,5 & 253,4 & 35,9 \\
\hline
\end{tabular}

Fonte: Setor de Custos da Gerência de Administração da SM SA-BH (2004).

Quadro 2. Demonstrativo do quantitativo de artigos (2005-2007) de acordo com as palavras-chave.

\begin{tabular}{|l|c|c|}
\hline \multicolumn{1}{|c|}{ Palavras- chave } & Medline & SciELO \\
\hline cost-effectiveness & 5185 & 9 \\
\hline cost-effectiveness and Brazil & 25 & 4 \\
\hline cost-effectiveness and Brazil and decision maker & 1 & 0 \\
\hline cost-effectiveness and decision maker & 112 & 0 \\
\hline cost-effectiveness and decision maker and health-care & 56 & 0 \\
\hline cost-effectiveness and decision maker and healthcare & 48 & 0 \\
\hline cost-effectiveness and decision maker and healthcare system & 42 & 0 \\
\hline cost-effectiveness and healthcare system & 1539 & 0 \\
\hline
\end{tabular}

Fonte: Base eletrônica de dados: M edlineeSciELO, 2005-2007. 
Dentreos artigos publicados, erelativos ao grupo de palavras-chave custo-efetividade/ gestor de sistema de saúde/ atenção à saúde, foi apropriado e sumarizado o conteúdo de seis estudos de relevantes autores que discutiram especificamentesobre o tema em questão ${ }^{11-16}$. Esses estudos, em grande medida, subsidiaram a reflexão.

De forma consensual, todos os autores citam a insuficiência do uso da ferramenta pelos gestores para a tomada de decisão. Assim como referem que, potencialmente, o problema está relacionado à inexistente padronização metodológica e à forma de apresentação dos resultados.

\section{Discussão econsiderações}

A popularização da avaliação econômica em saúde no mundo economicamente desenvolvido deveria servir como exemplo para que também houvesse a apreensão dessa prática no nosso meio. Principalmente porqueo Brasil, ao alocar um quantitativo reduzido de recursos na dimensão per capita, se coloca sob o efeito deletério da escassez de recursos e, nesta medida, necessita apreender este instrumental para dar eficiência à alocação dos recursos no setor.

O ProgramaSAM U/192, objeto de análise desta reflexão - até mesmo pelo seu alto custo -, se enquadra como uma das prioridades em termos de necessidade de avaliação econômica. Sendo que para a sua consecução, ou de quaisquer programas, é exigida a apreciação de uma série de pré requisitos.

Tomando o exemplo - Programa SAM U/192, que écomposto por um conjunto de intervenções, as quais podem ser analisadas separadamente por meio de uma análise de custo-efetividade ou do custo-consequência ou pela síntese do conjunto destas à luz do custo de oportunidade -, sua avaliação não prescinde, dentre a gama de pré-requisitos, do dimensionamento do(s) seu(s) objetivo(s) e suas categorias analíticas (variáveis) afins e de uma base de dados abrangente.

No caso então do Programa SAMU/192, que tem como objetivo prestar socorro à população, mediante ordenação de uma central médica de regulação deurgências, no intuito dereduzir o número deóbitos, o tempo de internação em hospitaise sequelas consequentes da falta de socorro precoce nos casos de natureza traumática, clínica, pediátrica, cirúrgica, gineco-obstétrica e de saúde mental ${ }^{17}$, é necessário coletar, ou ter acesso não só às informações referentes à atenção pré-hospitalar, mas também as da atenção hospitalar e pós-hospitalar.
E no que concerne a essa necessidade, inclusive para o balizamento da efetividade e a identificação do custo deste programa à luz da nossa realidade, os dados e/ou informações requeridas - de forma sucinta e sem a pretensão de esgotar todas as possibilidades -, seriam o cadastro do paciente, o diagnóstico (morbimortalidade), as intervenções terapêuticas realizadas (tendo como base protocolos de atenção) e o custo de cada ação prestada, o tempo decorrido entre 0 atendimento e a chegada ao hospital referenciado, os procedimentos realizados durantea hospitalização eseu valor ( preço) ou custo, o tempo de permanência no hospital, os procedimentos realizados na atenção pós-hospitalar (recuperação), a estimativa do custo desta atenção ao longo de um período de tempo (time preference) e informação referenteà qualidade de vida - normalmente obtida por meio da aplicação de questionários ou de outros instrumentos, a partir de métodos econométricos ou psicométricos.

Ainda na especificidade da avaliação da efetividade, é importante obter informações sobre 0 acesso do paciente à atenção hospitalar; ou seja, a busca de vaga nos hospitais de retaguarda. Essas informações são obtidas através de um sistema de regulação de leitos.

Sem dúvida, a fragmentação e pulverização da informação é um dos problemas para a condução de uma avaliação econômica, principalmente pela consequente debilidade dos resultados.

No entanto, o grande problema é que a especificação dos nossos bancos de dados não permite esse relacionamento. 0 que leva, por vezes, à necessidade de se desenvolver um sistema de informação relativo ao objeto sob análise ${ }^{18,19}$. Ou, como tem sido o praticado no nosso meio, utilizar apenas como subsídio para esse tipo de avaliação as evidências científicas advindas da literatura internacional, o que, conforme já explicitado pela literatura, incorre no problema da generalização (generalisability $)^{20}$.

0 problema apontado é decorrente da organização histórica das informações no Brasil - construída sob distintas perspectivas. N os anos oitenta ena perspectiva do M PAS/INAM PS (M inistério da Previdência eAssistência Social/I nstituto N acional deAssistência M édica ePrevidência Social), a construção ocorreu de forma centralizada e a partir de dadosfinanceiros; já, no mesmo período ena perspectiva do M inistério da Saúde, voltada para determinados agravos ou grupos de risco ${ }^{21}$.

Nos últimos anos, o M inistério da Saúde vem semobilizando no sentido de construir uma Política de Informação e Informática em saúde, a qual tem como objetivo o de integrar e articular as in- 
formações em saúde a partir das ações individuais e coletivas que possam subsidiar o conhecimento dos serviços e ações em saúde e do impacto desses na saúde da população22.

N estesentido, a proposição de cadastrosabrangentes, como, por exemplo, o Cartão SUS, permite, em certa medida, a promoção deste relacionamento ${ }^{23}$ e, por conseguinte, a condução de avaliações econômicas em nosso meio.

No que se refere à estrutura desta base cadastral, ela deve suprir as especificidades de cada área ou mesmo de cada nível de governo e, para tanto, estão disponíveis no mercado tecnologias apropriadas para este fim ${ }^{24}$.

M esmo que não faça parte do escopo deste estudo, mas apenas como um elemento complementar, e na perspectiva da investigação sobre tecnologias de informação, foi desenvolvido 0 ambiente Data Warehouse (DW) - coleção de tecnologias relacionada às necessidades gerenciais que objetiva apoiar o usuário de sistemas às tomadas de decisão ${ }^{25}$ - e, recentemente, inclusive no nosso meio, a formatação do Reclink, que relaciona de forma probabilística grandes bancos de dados a partir de variáveis afins, como, por exemplo, o nome, o CPF ou o número do cartão SU S26.

A importância destas tecnologias se assenta na possibilidade de integrar bases de dados heterogê neas dentro e externas à organização ${ }^{27-29}$.

0 conjunto de tecnologias de informação disponível é suficiente para atender os requisitos de uma análise de custo- efetividade. Entretanto, para que a sua incorporação nos sistemas de informação alcance esse objetivo, é imprescindível que os gestores promovam uma revisão conceitual nos seus processos de trabal ho, de modo a incorporar os conceitos definidos pelo paradigma dos sistemas de apoio à decisão - conjunto de dados baseado em assunto, integrado, não-volátil e variável em relação ao tempo ${ }^{30}$. Isto permitirá a especificação de sistemas de informações que, ao serem efetivamente implantados, capturem os dados necessários para subsidiar esse novo enfoque do processo decisório.

A condução adequada para uma avaliação econômica em saúde também pressupõe que fatores metodológicos sejam considerados, tais como a elaboração cuidadosa da questão-chave, a seleção adequada das alternativas sob comparação e a avaliação da incerteza, afeita ao setor (saúde), a qual nem sempre pode ser medida como o habitualmente realizado em relação a outras questões econômicas ${ }^{11-16}$.

Ainda no que concerneà condução, a proposição de uma metodologia de avaliação do tipo rá- pida, tal como desenvolvida para a avaliação do desempenho do sistema desaúde ${ }^{31}$, podeser o grande passo para a condução de estudos na esfera operacional de saúde. Principal mente pelo fato do nível operacional requerer agilidade na consecução de suas ações e também pelo fato de que hoje as instituições municipais de grande porte têm um número considerável de recursos humanos bem preparados, em termos acadêmicos, capazes de desenvolver estudos desta ordem.

$\mathrm{Na}$ especificidade da tomada de decisão pelos gestores, por meio de estudos de avaliação econômica, não se pode resumir o problema apenas à deficiência dos sistemas de informação no nosso meio. M as também devido aos estudos utilizarem, excessivamente, estimativas em detrimento de bases consolidadas de dados eà inadequação do processo de trabalho nas instituições para conduzir uma avaliação de boa qualidade. É importanteressaltar a necessidade de se adequar tomadas de decisão a partir da evidência advinda do resultado dos estudos, que muitas vezes são elaborados de forma a requerer um alto nível de compreensão ${ }^{12,32,33}$. Vale lembrar queno Brasil - país de grande dimensão geográfica - existem distintas situações socioeconômicas e demográficas, as quais também geram problemas de generalização.

Talvez por este e outros problemas, autores como Gafni e Birch não considerem o instrumento, particularmente no seu conteúdo incremental da avaliação, como o melhor método para tomada de deci são ${ }^{34}$, na medida em que a análise incremental nem sempre incorpora o contexto sob o qual o gestor se depara.

N esta mesma linha de pensamento, $M$ ays et al. fazem uma crítica em relação às sínteses de evi dências ao referir que as sínteses não são suficientes para determinados problemas, sugerindo, inclusive, que outras fontes e métodos sejam identificados para a síntese de evidências ${ }^{35}$.

Já outros autores, mesmo reconhecendo que o instrumental da avaliação econômica tem problemas, argumentam, de forma positiva e no sentido de minimizá-los, os seguintes pontos: a disponibilidade de vários métodos de microsimulação para a modelagem de análise de decisão, a partir do avanço da tecnologia computacional11; ; a supressão de itens de custos não relevantes ${ }^{36}$ ea absorção pelo público alvo do uso da ferramenta para efeito de decisão gerencial no setor saúde ${ }^{13}$.

Dentre os argumentos, o mais intrigante é 0 que se refere à supressão de itens de custo, em vista de que para os gestores de sistemas de saúde a perspectiva ideal, e a mais simples, de condução de um estudo é a do governo, ou seja, aquela que inclui 
apenas os custos diretos e não os da sociedade. A perspectiva da sociedade não só quantifica evalora os custos diretos, mas também os custos indiretos e, em al guns casos, também os intangíveis.

Certamente, o cenário atual não é favorável à condução e/ou à apreensão de estudos sobreavaliação econômica pelos gestores de nível local. Mas, para que se logre o objetivo de efetivar essas avaliações na área da saúde nos municípios de grande porte, eparticularmenteno nível operacional deatenção à saúde, é fundamental realizar um relacionamento entre os vários bancos de dados nacionais, os específicos institucionais e outros de interesse.

Assim como é imperativo que os gestores nas distintas esferas de governo, à luz do já disposto em relação à integração de sistemas nacionais sujeita às especificidades locais, invistam no desenvolvimento de sistemas de informações. Para que, através destes, não só se reconheçam as necessidades em saúde da população sob sua responsabilidade, mas que também propiciem uma avaliação sobre a efetividade e o custo-efetividade das intervenções e programas que realmente respondam a essas necessidades. Sendo, no entanto, fundamental ter sempre como uma prerrogativa 0 (re)conhecimento do contexto sob o qual essas intervenções e programas estão sendo implementados, e que, fundamentalmente, se "sirvam" de estudos desta ordem como um importante aporte para as suas tomadas de decisão.

\section{Colaboradores}

LG Sancho trabal hou na concepção teórica, elaboração e redação final do texto e JM C Vargens trabalhou na elaboração do texto.

\section{Referências}

1. ANVISA. Boletim Brasileiro de Avaliação de Tecnologias em Saúde A T S G, 2006. [acessado 2007 out 21]. Disponível em: http://www.bvsms.saude.gov.br/bvs/ct/ pdf/brats2006_n2.pdf

2. Garber AM. Advances in cost-effectiveness analysis of health interventions. Cambridge: National Bureau of Economic Research; 1999. [N BER Working Paper 7198]

3. Vianna CM M, Caetano R. Diretrizes metodológicas para estudos de avaliação econômica de tecnologias para 0 M inistério da Saúde. [acessado 2007 out 06]. Disponível em: http://portal.saude.gov.br/portal/arquivos/ pdf/diretrizes metodologias ave.pdf

4. Brasil. Ministério da Saúde. Avaliação econômica em saúde: desafios para a gestão no Sistema Ú nico de Saúde. [acessado 2008 fev 22]. Disponível em: http://portal. saude.gov.br/portal/arquivos/pdf/livro_aval_econom_ saude.pdf

5. Martins E. Contabilidade de custos. São Paulo: Atlas; 1994.

6. Varian HR. Microeconomia: princípios básicos. Rio de Janeiro: Campus; 1994.

7. Mandelblatt JS, Fryback DG, Weintein MC, Russel LB, Gold M R, Hardon DC. Assessing the Effectiveness of Health Interventions. In: Gold M R, Siegel JE, Russell LB, Weinstein MC. Cost-Effectiveness in Health and Medicine. New York: Oxford University Press; 1996. p. 135-168.

8. Caetano R,Vianna CM M. Custo-efetividade no rastreamento do câncer cérvico-uterino no Brasil: um estudo exploratório. [acessado 2006 dez 17]. Disponível em: http://www.inca.gov.br

9. Drummond MF, O'Brien BJ, Stoddart G. M ethods for Economic Evaluation of $\mathrm{H}$ ealth Care Programmes. N ew York: Oxford University Press; 1997. 
10. Gold, MR, Siegel JE, Russell LB, Weinstein MC. CostEffectiveness in $\mathrm{H}$ ealth and Medicine. New York: Oxford University Press; 1996.

11. Drummond MF, Aguiar-Ibanez R, Nixon J. Economic evaluation. Singapore M ed J. 2006; 47(6):456-461.

12. Iglesias CP, Drummond M F, Rovira J, NEVALAT Project Group. Health-care decision-making processes in Latin America: problems and prospects for the use of economic evaluation. Int J Technol Assess Health Care 2005; 21(1):1-14.

13. Gold M R, Franks P, Siegelberg T, Sofaer S. Does providing cost-effectiveness information change coverage priorities for citizens acting as social decision makers? H ealth Policy 2007; 83(1):65-72.

14. Birch S, Gafni A. Information created to evade reality (ICER): things we should not look to for answers. Pharmacoeconomics 2006; 24(11):1121-1131.

15. Torrance GW. Utility measurement in healthcare: the things I never got to. Pharmacoeconomics 2006; 24(11):1069-1078.

16. Luce BR. What will it take to make cost-effectiveness analysis acceptable in the U nited States? M ed Care 2005; 43(Supl. 7):44-48.

17. Brasil. M inistério da Saúde. 0 que é SAM U ? [acessado 2005 ago 11]. Disponível em: http://wwww.saude. gov.br

18. Sesso R. Inquérito epidemiológico em unidades de diálise do Brasil. J. Bras. N efrol 2000; 22 (Supl. 2):23-26.

19. Silva LK. Technology assessment of different levels of neonatal care 1984-1992 [thesis]. Birmingham: Faculty of Commerce and Social Sciences, University of Birmingham; 1992.

20. Ade AE, Claxton K, Sculpher M. Evidence synthesis, parameter correlation and probabilistic sensitivity analysis. Health Econ. 2006; 15:373-381.

21. Carvalho DM. Sistemas de Informação e Alocação de Recursos: um estudo sobre as possibilidades de uso das grandes bases de dados nacionais para uma alocação orientada de recursos [dissertação]. Rio de Janeiro (RJ). Instituto de M edicina Social, Universidade do Estado do Rio de Janeiro; 1998.

22. Brasil. M inistério da Saúde. A Construção da Política Nacional de Informação e Informática em Saúde 2003. Versão 1.3 resumida. [acessado 2005 set 08]. Disponível em http://www.datasus.gov.br

23. Bittencourt AS, Camacho LAB, Leal M C. O Sistema de Informação Hospitalar e sua aplicação na saúde coletiva. Cad Saude Publica 2006; 22(1):19-30.

24. ABRASCO. Compatibilização de bases de dados Nacionais. Relatório da Oficina de trabalho realizada no $\mathrm{V}$ Congresso Brasileiro de Saúde Coletiva eV Congresso Paulista de Saúde Coletiva 1997. [acessado 2005 out 20]. Disponível em: http://portal.saude.gov.br/portal/arquivos/ pdf/iesus_vol7_3_descentralizacao.pdf
25. Jarke M, Vassiliou Y. Data Warehouse Quality: A Review of DWQ Project. $2^{\text {nd }}$ Conference on Information Quality. Massachussetts Institute of Technology, Cambridge 1997. [acessado 2005 out 29]. Disponível em: http://www.cs.brown.edu

26. Coeli CM , Camargo Jr KR. Avaliação de diferentes estratégias de blocagem no relacionamento probabilísticos de registros. Rev. Bras. Epidemiologia 2002; 5:185-196.

27. Afilalo M, Lang E, Boivin JF. The Impact of a Standardized Information System Between the Emergency Department and the Primary Care N etwork: Effects on Continuity and Quality of Care. Ottawa: Canadian Health Research Foundation; 2003.

28. Green R, Joyce P. Data Warehouse - Unlocking our Health Service's Data. [acessado 2005 out 26]. Disponível em: http://www.himaa.org.au

29. Winker WE, Hidiroglou M. Developing Analytic Programming Capability to Empower Organization. [acessado 2005 out 27]. Disponível em: http://www.census.gov/

30. Inmon WH. Como Construir o Data Warehouse. Rio de Janeiro: Campus; 1997.

31. Almeida C, Macinko J. Validação de uma metodologia de avaliação rápida das características organizacionais e do desempenho dos serviços de atenção básica do Sistema único de Saúde (SUS) em nível local. [acessado 2005 out 13]. Disponível em: http://www.opas.org.br/servico/ arquivos/Sala5558.pdf

32. Drummond $M$, Sculpher M. Common methodological flaws in economic evaluations. M ed Care 2005; 43(Supl. 7):5-17.

33. Weinstein MC. Recent development in decision-analytic modelling for economic evaluation. Pharmaeconomics 2006; 24(11):1043-1053.

34. Birch S, Gafni A. The biggest bang for the buck or bigger bucks for the bang: the fallacy of the costeffectiveness threshold. J Health Ser Res Policy 2006; 11(1):46-51.

35. M ays N, Pope C, Popay J. Systematically reviewing qualitative and quantitative evidence to inform management and policy-making in the health field. J H ealth Ser Res Policy 2005; 10 (Supl. 1):6-20.

36. Brouwer WB, Van Exel NJ, Baltussen RM, Rutten FF. A dollar is a dollar is a dollar- or is it? Value $\mathrm{Health}$ 2006; 9(5):341-347.

Artigo apresentado em 17/03/2007

Aprovado em 15/02/2008

Versão final apresentada em 29/02/2008 